\title{
Prediction of gas production kinetics of maize stover and ear by near infrared reflectance spectroscopy*
}

\author{
S. Kruse, A. Herrmann'1, R. Loges and F. Taube \\ Institute of Crop Science and Plant Breeding, Grass and Forage Science/Organic Agriculture, \\ Christian-Albrechts-University Kiel \\ Olshausenstr. 40, D-24098 Kiel, Germany
}

(Received 30 November 2007; revised version 4 April 2008; accepted 24 June 2008)

\begin{abstract}
This study was conducted to evaluate the potential of NIRS to predict gas production (GP) kinetics for maize ear and stover. Two approaches were applied to characterize GP kinetics: 1. describing GP profiles by fitting an exponential model and 2. by calculating GP for various incubation intervals. In the first approach, NIRS prediction equations could explain a high proportion of variability related to the fermentation rate $(c)$, the asymptotic GP $(A)$, and the lag phase $(L)$ of ear samples in the calibration subset $\left(\mathrm{R}^{2}: 0.81-0.87\right)$, while for stover predictive ability was inferior. Validation showed satisfactory results only for the fermentation rate $\left(\mathrm{R}^{2}: 0.81-0.88\right)$. In the second approach NIRS calibration was acceptable for GP in several incubation intervals, but validation showed satisfactory results for intervals $3-7,7-12$, and 7-16 h of stover samples only $\left(\mathrm{R}^{2}>0.74\right)$. Further research is required to elucidate potential sources of error before obtaining robust NIRS equations.
\end{abstract}

KEY WORDS: gas production, incubation interval, NIRS, maize silage, ear, maize stover

\section{INTRODUCTION}

The possibility to accurately predict the nutritive value of forage crops is a prerequisite for designing rations based on the animal's requirements, but also for

\footnotetext{
* Supported by the Ministry of Economics and Labor through the German Federation of Industrial Research Associations "Otto von Guericke"

${ }^{1}$ Corresponding author: e-mail: aherrmann@email.uni-kiel.de
} 
directing forage crop breeding. The most precise determination of a crop's nutritive value derives from in vivo feeding studies. Routine analysis, however, is hampered by the need of laboratory facilities and cannulated animals, large quantities of feed and high costs. To this account, in vitro techniques were developed. The in vitro gas production (GP) technique (Menke et al., 1979) is a simple and robust method, based on the close relationship between gas produced from anaerobic fermentation with rumen liquor and feed degradation, which allows to estimate various forage quality characteristics. It has some advantages over in vitro methods that are based on measuring fermentation residues, since for instance, not only information on the absolute extent of feed digestion is provided, but also on the degradation kinetics (Getachew et al., 2004). The efficiency of sample evaluation brought about by automation (Theodorou et al., 1994) makes the GP technique applicable to plant breeding programs (Getachew et al., 2005). Nevertheless, the time need for incubation and the necessity of rumen fluid donor animals is a disadvantage. This obstacle might be overcome by the near infrared reflectance spectroscopy (NIRS) technology, which has shown great potential to predict forage quality parameters in a rapid and non-destructive way (Stuth et al., 2003). For forage grasses, NIRS gave promising results in predicting static gas volumes, while for kinetic parameters results were less accurate (Herrero et al., 1997; Andrés et al., 2005). With respect to maize silage, NIRS prediction of fermentation kinetics has shown only moderate success so far (Lovett et al., 2004). This inability has primarily been attributed to the inhomogeneity of maize, which represents a mixture of concentrate and roughage, i.e. a high starch content in grain and mostly fibrous components in the stover.

The objective of this study therefore was to explore the potential of NIRS for predicting GP kinetics separately for ear and stover. This is not only interesting from a scientific point of view, but also has practical relevance, because maize stover is an important ruminant feed in many small-holder crop-livestock production systems of developing countries and maize-cob-mix is a common concentrate in intensive cattle production.

\section{MATERIAL AND METHODS}

\section{Feed samples}

The study is based on data collected in a 3-year (2001-2003) field experiment conducted at an experimental farm of the University of Kiel, northern Germany. Eight silage maize varieties covering a wide range of maturity groups (early to mid-late) and different maturation types were sown in early May. For further details concerning the crop management see Kruse et al. (2008). To obtain a broad sample population, crop samples were taken on six dates, which were chosen to 
be in line with developmental stages of a mid-early reference hybrid, scheduled to phenological stage of BBCH 32 (Meier, 2001), and ear dry matter contents of $20,30,40,50$ and $55 \%$. On each sampling date, ten plants per plot were harvested by hand clipping. The plants were weighed, separated into ear and stover (including husks), and chopped. Representative sub-samples were oven-dried at $105^{\circ} \mathrm{C}$ to determine dry matter content. Another sub-sample of both ear and stover was stored at $-18^{\circ} \mathrm{C}$ for forage quality determination. After freeze-drying, the samples were ground in a Cyclotec mill (Foss Tecator AB, Sweden) to pass a $1 \mathrm{~mm}$ sieve. In total, 480 samples of ears and 640 samples of stover were available for NIRS subset selection and subsequent determination of gas production and chemical composition.

\section{Chemical analysis}

Neutral detergent fibre (NDF), acid detergent fibre (ADF) and acid detergent lignin (ADL) were determined according to Goering and van Soest (1970; cited in Naumann and Basler, 1976), where ear samples were pre-treated with heat-stable amylase to ensure starch degradation. Nitrogen was determined in a $\mathrm{CN}$-analyser (elementar, vario Max CN; Hanau, Germany) and multiplied by 6.25 to obtain crude protein (CP). Starch was analysed on the basis of an enzymatic method by Brandt et al. (1987), and water soluble carbohydrates (WSC) according to a modified anthrone method as described by van Handel (1967) and McAllen (1985).

\section{Gas production}

The in vitro gas production was analysed according to Menke and Steingass (1988). Approximately $200 \mathrm{mg}$ of each sample was placed in a glass syringe. Buffer mineral solution was prepared along with modifications proposed by Liu et al. (2002). The solution was placed in a water bath at $39^{\circ} \mathrm{C}$ under continuous $\mathrm{CO}_{2}$ flushing. Rumen fluid was obtained from two ruminally fistulated German Red Pieds steers before morning feeding. The steers received a mixed diet of perennial ryegrass hay and concentrates $(2: 1, \mathrm{wt} / \mathrm{wt})$. The rumen fluid taken from both animals was mixed, filtered through cheesecloth and flushed with $\mathrm{CO}_{2}$, as all laboratory handlings of rumen fluid were carried out. Subsequently, the rumen fluid was added to the buffered mineral solution $(1: 2, \mathrm{v} / \mathrm{v})$ and mixed. Thirty $\mathrm{ml}$ of the incubation medium were pipetted into each syringe, which was placed in a rotor $(1 \mathrm{rpm})$ within an incubator at $39^{\circ} \mathrm{C}$. Three blanks containing only $30 \mathrm{ml}$ of medium were included in each assay, as were triplicates of standard hay and standard concentrate obtained from the Institute of Animal Nutrition, Hohenheim University (Germany). Gas production was recorded after 1, 3, 5, 7, 12, 16, 24, 48 and $72 \mathrm{~h}$ of incubation. The runs were replicated three times. Fermentation 
kinetics were characterized by fitting the data to the model of France et al. (2000) to derive curve parameters, which then were predicted by NIRS. This common approach involves two errors, one related to curve fitting and one resulting from NIRS calibration. We therefore applied an additional approach to circumvent the curve fitting error by characterizing the fermentation dynamics by the gas volume produced during specific intervals $(3-5,3-7,5-12,7-12,5-16,7-16,12-16,12-24$, 24-72 and 16-72 h), which may be related to the degradation of specific chemical components (Cone et al., 1994; Chai et al., 2004).

\section{Near infrared reflectance spectroscopy of gas production kinetics}

NIRS prediction equations were developed separately for ear and stover for (i) the curve parameters of the model proposed by France et al. (2000), i.e. parameter $A$ (asymptotic gas production, $\mathrm{ml}$ ), $c$ (fractional rate of fermentation, $\mathrm{h}^{-1}$ ), and $L$ (lag time, h), and (ii) the gas produced in 10 different incubation intervals (3-5, $3-7,5-12,7-12,5-16,7-16,12-16,12-24,24-72$ and 16-72 $\mathrm{h}$ ). To this end, all crop samples available were scanned with two replicates using a NIRSystems 5000 scanning monochromator (FOSS GmbH, Germany). Mathematical treatment of the spectra was performed using the ISI-NIRS2 Version 3.1 software (Infrasoft International ${ }^{\circledR}$, Port Matilda, PA, USA). Absorbance was recorded as $\log (1 / \mathrm{reflec}-$ tance $)=\log (1 / \mathrm{R})$ at $2 \mathrm{~nm}$ intervals throughout the near-infrared region $(1100$ $2500 \mathrm{~nm}$ ) to give a total of 700 data points. Subsequently, samples were checked for erroneous measurements and outliers, using the option 'centre samples', which provides a ranking of the spectral data on the basis of the standardized Mahalanobis distance $(\mathrm{H})$ from the average spectrum. The sample population boundary was defined $\mathrm{H}=3$ and outliers were removed.

Samples were chosen to represent the whole spectral and chemical variability of the sample population in the calibration and validation subsets, based on the pooled 2001 and 2002 data, and extended by the 2003 samples. The H-value was used as a criterion for selecting those samples in the population as being more variable on the basis of spectra features. The option 'select samples' on the basis of $\mathrm{H}$-value 0.6 was used to select calibration subsets representing the whole sample population, while the validation subsets were randomly selected after ranking the spectral data according to their $\mathrm{H}$ distance. A total of 88 representative samples of ear and 210 samples of stover were selected for measuring and calibrating gas production kinetics. For validation, 53 samples of each ear and stover were selected. The final number of samples included in NIRS analysis was variable due to missing values and outliers eliminated during the mathematical calibration process (Tables 2, 3 and 5).

Parameters in the following mathematical processing creating the predictive equations were sought to minimize the standard errors of calibration (SEC) and to 
maximize the coefficients of determination $\left(\mathrm{R}^{2}\right)$ using the Modified Partial LeastSquares (MPLS) method (Shenk and Westerhaus, 1991). The minimum F statistics for terms included in the equation was 8.0. Spectral data was analysed using different mathematical treatments as provided in Table 1. Pearson's correlation coefficient was computed to quantify the relation between chemical composition and gas volume produced during various incubation intervals.

Table 1. Mathematical treatments for calibrating gas production kinetics with approach I (parameters $A, c, L$ according to model by France et al., 2000), and approach II (gas production in different incubation intervals)

\begin{tabular}{|c|c|c|c|c|}
\hline \multirow[t]{2}{*}{ Item } & \multicolumn{2}{|c|}{$\begin{array}{l}\text { Mathematical } \\
\text { treatments } 1,2,3,4\end{array}$} & \multicolumn{2}{|c|}{$\begin{array}{l}\text { Scatter correction for } \\
\text { particle size }\end{array}$} \\
\hline & ear & stover & ear & stover \\
\hline \multicolumn{5}{|c|}{ Approach I } \\
\hline A & $2,4,4,1$ & $1,3,3,1$ & SNV/detrend & SNV/detrend \\
\hline$c$ & $1,3,3,1$ & $1,3,3,1$ & SNV/detrend & SNV/detrend \\
\hline$L$ & $1,3,3,1$ & $2,3,3,1$ & SNV/detrend & --- \\
\hline \multicolumn{5}{|c|}{$\begin{array}{l}\text { Approach II } \\
\text { incubation interval, } \mathrm{h}\end{array}$} \\
\hline $3-5$ & $1,3,3,1$ & $2,4,4,1$ & SNV/detrend & $\mathrm{SNV} /$ detrend \\
\hline $3-7$ & $1,5,5,1$ & $2,4,4,1$ & --- & --- \\
\hline $5-12$ & $2,3,3,1$ & $2,4,4,1$ & SNV/detrend & --- \\
\hline $7-12$ & $2,5,5,1$ & $2,4,4,1$ & SNV/detrend & --- \\
\hline $5-16$ & $2,5,5,1$ & $2,4,4,1$ & SNV/detrend & --- \\
\hline $7-16$ & $2,5,5,1$ & $2,4,4,1$ & --- & --- \\
\hline $12-16$ & $2,2,2,1$ & $2,4,4,1$ & SNV/detrend & --- \\
\hline $12-24$ & $2,4,4,1$ & $1,4,4,1$ & SNV/detrend & --- \\
\hline $24-72$ & $2,4,4,1$ & $1,4,4,1$ & $\mathrm{SNV} /$ detrend & --- \\
\hline $16-72$ & $2,2,2,1$ & $2,5,5,1$ & SNV/detrend & SNV/detrend \\
\hline
\end{tabular}

${ }^{1}$ number of derivate of the $\log (1 / R)$ spectrum; ${ }^{2}$ segment of the gap over which the derivative was calculated; ${ }^{3}$ number of data points used during first smoothing of the spectrum; ${ }^{4}$ number of data points used during second smoothing of the spectrum

\section{RESULTS}

Gas production kinetics from curve fitting. Due to sequential harvesting, samples were available over a wide range of maturity stages, with DM content varying between 156 to $571 \mathrm{~g} \mathrm{~kg}^{-1} \mathrm{DM}$ for ear and between 96 and $303 \mathrm{~g} \mathrm{~kg}^{-1} \mathrm{DM}$ for stover. This was reflected in the large variation of the laboratory determined kinetic parameters, which for instance ranged between 0.03 and $0.13 \mathrm{~h}^{-1}$ for the fractional fermentation rate (c) of the ear calibration subset (Table 2). Differences in kinetic 

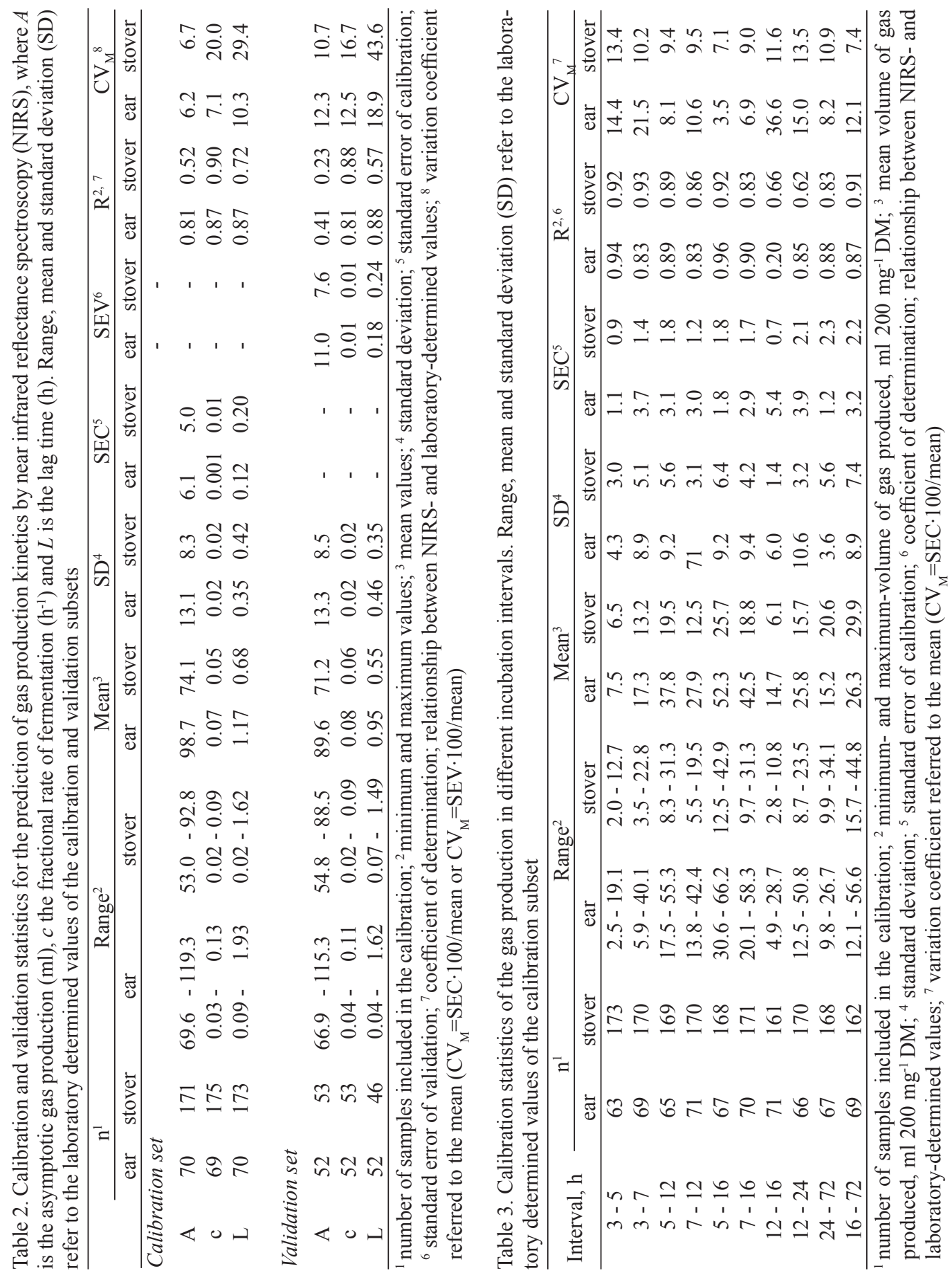
parameters between ear and stover were most apparent for the lag phase (L). NIRS calibration demonstrated satisfactory ability $\left(\mathrm{R}^{2}>0.81\right)$ to predict gas production parameters for ear samples, but was inferior for the stover as indicated by lower $\mathrm{R}^{2}$ for asymptotic GP (A) and lag phase (L), and higher standard errors of calibration (SEC) for the fermentation rate (c) and lag phase (Table 2). However, the SEC as a absolute, difference based statistic has only limited suitability for comparing the accuracy of NIRS prediction across populations, if those are characterized by large differences in GP characteristics, as for instance ear and stover, which differ substantially in the overall level of gas production and fermentation rates. We therefore additionally provided the variation coefficient referring to the mean $\left(C V_{M}=S E C \cdot 100 /\right.$ mean $)$, which should not exceed $10 \%$ of the mean determined by the reference method (Hruschka, 1987). $\mathrm{CV}_{\mathrm{M}}$-values basically confirm the lower predictive ability for stover. The accuracy of prediction achieved in validation pointed out difficulties with respect to the asymptotic GP and lag phase for both ear and stover, while fermentation rate could be satisfactorily predicted. The mean values, ranges and standard deviations were in a similar range for the calibration and validation set, indicating that the sample sets were comparable.

Gas production in incubation intervals. In addition to the curve fitting approach, which showed only moderate success so far, we investigated the potential to predict the GP in different incubation intervals. This is based on the assumption that the GP in specific intervals is closely related to the degradation of specific chemical constituents (Cone et al., 1994; Chai et al., 2004). Incubation of ear samples resulted in laboratory determined gas volumes ranging between $2.5 \mathrm{ml} 200 \mathrm{mg}^{-1}$ DM (3-5 h) and $66.2 \mathrm{ml} 200 \mathrm{mg}^{-1} \mathrm{DM}$ (5-16 h), (Table 3). Stover was characterized by a substantially lower gas production. The relationship between GP recorded in different intervals and chemical composition was described by correlation coefficients provided in Table 4. Gas produced in intervals 3-5, 3-7 and 12-16 h of ear samples was strongly related to WSC, CP, starch, cellulose and hemicellulose, whereas in stover relevant relationships were detected for intervals 3-5, 3-7, 16-72 and 24-72 h. ADL content seems to have no impact on GP in ear, while high stover ADL reduced GP in early incubation and increased GP later on.

The accuracy of prediction can be assessed from statistics provided in Tables 3 and 5. NIRS calibration was mostly acceptable for intervals 3-5, 3-7 (stover), 5-12, 7-12, 5-16, 7-16, 24-72 and 16-72 h with low SEC, CV-values around or below $10 \%$, and coefficients of determination exceeding 0.83 . For intervals 3-7 (ear), 12-16, and 12-24 h either $\mathrm{R}^{2}$ - or CV-values were noticeably poorer. In contrast to calibration, validation statistics demonstrate severe shortcomings. With the exception of intervals 3-7, 5-12, 7-12, 5-16 and 7-16 h of stover incubation, the calibration equations failed to accurately estimate GP, as indicated by $\mathrm{R}^{2}$-values below 0.69 and/or SEV exceeding $10 \%$ of the mean. 

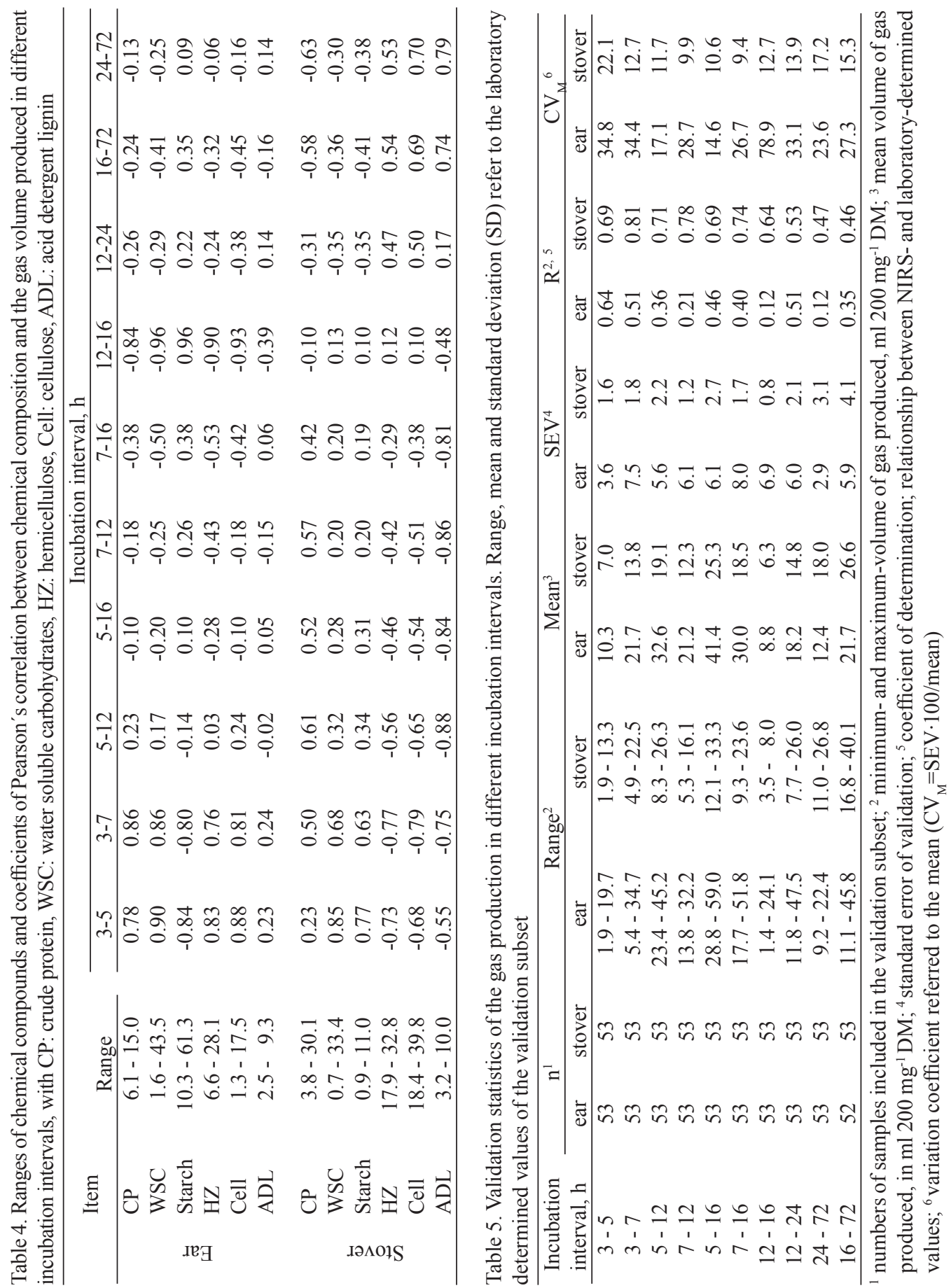


\section{DISCUSSION}

A comparison of the derived kinetic parameters with published values is difficult due to the limited number of studies available, which furthermore differ in crop maturity stage, sample processing, incubation length, and fitted models. Fermentation of ear resulted in higher total GP (asymptote) compared to values reported by DePeters et al. (2007) for grain. Ear fermentation rate was consistent with the study by Carro and Ranilla (2003) on grains. For stover, we detected a higher asymptotic value than Tolera and Sundstøl (1999), probably due to an earlier harvest in our trials. Fermentation rates, however, were in agreement. Compared to the work by Tang et al. (2006) on grain maize substantial differences became evident for all parameters. With respect to NIRS prediction of GP dynamics, the results of the present study document an insufficient performance for both approaches investigated. Consistent to the study of Lovett et al. (2004) on maize silage, prediction of the asymptotic GP was inferior compared to the fermentation rate and lag phase.

Several factors may influence the success of NIRS equation development, such as the accuracy of laboratory determined data and the errors related to NIRS calibration. Crucial to improving NIRS predictions is the degree of accuracy associated with the reference method, since the validity of NIRS-predicted data will never be better than the reference methods used for establishing the NIRS equations. Parameters measured by biological methods such as the gas production technique are subject to a multitude of error sources, which may increase variability.

With respect to the accuracy of gas production measurements, the repeatability within a run and the reproducibility between runs on different days have to be considered. While Getachew et al. (2002) reported a high degree of reproducibility, Van Laar et al. (2006) pointed out considerable differences among incubation runs. Pell and Schofield (1993), evaluating the variation of measurements over incubation time, detected a slight decline in repeatability. In the present study, however, no clear trend in prediction accuracy over incubation time became evident.

Another potential reason for poor NIRS statistics refers to unexplained or systematic errors (bias), which may be introduced when NIRS equations are used that are not properly represented in the calibration data set. This does not apply to the present study, where calibration and validation subsets originated from the same field experiment. It may therefore be assumed that all sources of variation encountered in data sampling and routine analysis were covered in the calibration as well as in the validation subsets. With respect to the curve fitting approach, however, a 72-h incubation period possibly was not long enough to ensure an accurate estimation of the asymptotic GP.

It is usually assumed that the calibration and validation subsets should represent the range of expected variation in the desired trait, i.e. a high degree of inho- 
mogeneity with respect to chemical composition of the subsets is advantageous (Stuth et al., 2003). Lovett et al. (2004), however, studying the ability to predict GP kinetics of maize silage, identified the inhomogeneity of maize samples, which contain both fibrous and starchy fractions, as a main error source in prediction performance of NIRS. It is well known that feeds rich in non-structural carbohydrates produce a lower acetate/propionate ratio compared to feeds rich in fibre, resulting in lower yields of gas volumes (Beuvink and Spoelstra, 1992). Lovett et al. (2004) regard this indirect effect of chemical composition on the short chain fatty acids (SCFA) profile as the main cause for the inability of NIRS to predict GP kinetics. Obviously, this effect also accounts for the results of our study. Although the crop was separated into ear and stover, the wide range of maturity stages has caused a substantial inhomogeneity in chemical composition within each group (Table 4). In the case of the ear, the ratio of vitreous to floury endosperm, which increases with advancing maturity and reduces ruminal starch availability (DePeters et al., 2007), may have affected the SCFA profile and GP. Moreover, most plant carbohydrate types absorb in similar spectral regions (Deaville and Givens, 1998), which also may limit NIRS prediction of GP for different incubation intervals.

These assumptions are supported by the results of the GP dynamics detected in different incubation intervals (Tables 3 and 5). Prediction of GP was successful $\left(\mathrm{R}^{2} \geq 0.74\right)$ only for intervals $3-7,7-12$ and $7-16 \mathrm{~h}$ of stover samples, for which it may be assumed that large part of the GP resulted from degradation of one chemical constituent. With respect to interval 3-7 h, the coefficient of correlation indicates that mainly WSC were fermented, since stover starch content is low. Easily fermentable cell wall is known to be degraded within 5-15 h incubation (Cone et al., 1994). Afterwards, less fermentable cell wall and microbial turnover are the main drivers of GP (Cone et al., 1994), as indicated by rising R-values for cellulose and hemicellulose with incubation time. In ear samples, overlapping fermentation of various compounds, as for instance CP and WSC in early incubation, may have hampered NIRS prediction. Coefficients of correlation, however, have to be interpreted with care as spurious correlations between fibre fractions and GP may occur, caused by maturity acting as lurking variable.

\section{CONCLUSIONS}

The use of near-infrared reflectance spectroscopy (NIRS) for predicting gas production kinetics of maize silage has thus far been of limited validity, which was primarily ascribed to the inhomogeneity in substrate composition. Our attempts to increase homogeneity by developing calibration equations separately for ear and stover showed promising results for NIRS calibration, while validation statistics were not fully convincing. For stover, predictive ability by the curve fitting approach 
might be enhanced with longer incubation time. Altogether, NIRS prediction of GP kinetics remains a considerable challenge. On the one hand, a large variation in sample population is required in order to obtain representative calibrations. On the other hand, a wide variation in substrate composition and degradability, as obtained in our study by differences in crop developmental stage, seems to be a chief cause for the limited potential of NIRS to predict GP kinetics.

\section{ACKNOWLEDGEMENTS}

We thank M. Gierus and K.-H. Südekum for helpful discussions. We acknowledge C. Beckmann for conducting part of the gas production measurements. We also wish to thank W. Kühl for her expert technical assistance.

\section{REFERENCES}

Andrés S., Calleja A., López S., González J.S., Rodríguez P.L., Giráldez F.J., 2005. Prediction of gas production kinetic parameters of forages by chemical composition and near infrared reflectance spectroscopy. Anim. Feed Sci. Tech. 123-124, 487-499

Beuvink J.M.W., Spoelstra S.F., Hogendorp R.J., 1992. An automated method for measuring timecourse of gas production of feedstuffs incubated with buffered rumen fluid. Neth. J. Agr. Sci. 40, 401-407

Brandt M., Schuldt A., Mannerkorpi P., Vearasilp T., 1987. Zur enzymatischen Stärkebestimmung im Darminhalt und Kot von Kühen mit hitzestabiler Amylase. Arch. Anim. Nutr. 37, 455

Carro M.D., Ranilla M.J., 2003. Effect of the addition of malate on in vitro rumen fermentation of cereal grains. Brit. J. Nutr. 89, 181-188

Chai W.Z., van Gelder A.H., Cone J.W., 2004. Relationship between gas production and starch degradation in feed samples. Anim. Feed Sci. Tech. 114, 195-204

Cone J.W., van Gelder A.H., Veerman E.T., van Vuuren A.M., 1994. In vitro estimation of rumen fermentable organic matter using rumen fluid and a cell free preparation of rumen fluid. Neth. J. Agr. Sci. 42, 343-356

Deaville E.R., Givens D.I., 1998. Regions of normalised near infrared reflectance difference spectra related to the rumen degradation of fresh grass, grass silage and maize silage. Anim. Feed Sci. Tech. 72, 41-51

DePeters E.J., Getachew G., Fadel J.G., Corona L., Zinn R.A., 2007. Influence of corn hybrid, protease and methods of processing on in vitro gas production. Anim. Feed Sci. Tech. 135, $157-175$

France J., Dijkstra J., Dhanoa M.S., López S., Bannik A., 2000. Estimating the extent of degradation of ruminant feeds in vivo from a description of their gas production profiles observed in vitro. 1 . Derivation of models and other mathematical considerations. Brit. J. Nutr. 83, 143-150

Getachew G., Crovetto G.M., Fodevila M., Krishnamoorthy U., Sigh B., Spanghero M., Steingass H., Robinson P.H., Kailas M.M., 2002. Laboratory variation of $24 \mathrm{~h}$ in vitro gas production and estimated metabolizable energy values of ruminant feeds. Anim. Feed Sci. Tech. 102, 171-182

Getachew G., DePeters E.J., Robinson P.H., Fadel J.G., 2005. Use of an in vitro gas production technique to evaluate microbial fermentation of ruminant feeds and its impact on fermentation products. Anim. Feed Sci. Tech. 123-124, 547-559 
Getachew G., Robinson P.H., DePeters E.J., Taylor S.J., 2004. Relationships between chemical composition, dry matter degradation and in vitro gas production of several ruminant feeds. Anim. Feed Sci. Tech. 111, 57-71

Handel van E., 1967. Determination of fructose and fructose-yielding carbohydrates with cold anthrone. Anal. Biochem. 19, 193-194

Herrero M., Jessop N.S., Fawcett R.H., Murray I., Dent J.B., 1997. Prediction of the in vitro gas production dynamics of kikuyu grass by near infrared reflectance spectroscopy using spectrally structured sample populations. Anim. Feed Sci. Tech. 69, 281-287

Hruschka W.R., 1987. Data analysis: wavelength selection methods. In: P. Williams, K. Norris (Editors). Near Infrared Technology in the Agricultural and Food Industries. American Association of Cereal Chemists, Inc. St. Paul, Minnesota (USA)

Kruse S., Herrmann A., Kornher A., Taube F., 2008. Evaluation of genotype and environmental variation in fibre content of silage maize using a model-assisted approach. Eur. J. Agron. 28, 210-223

Liu J.X., Susenbeth A., Südekum K.-H., 2002. In vitro gas production measurements to evaluate interactions between untreated and chemically treated rice straws, grass hay and mulberry leaves. J. Anim. Sci. 80, 517-524

Lovett D.K., Deaville E.R., Mould F.L., Givens D.I., Owen E., 2004. Using near infrared reflectance spectroscopy (NIRS) to predict the biological parameters of maize silage. Anim. Feed Sci. Tech. $115,179-187$

Mc Allan A.B., 1985. Analysis of carbohydrate in the alimentary tract and its nutritional significance. In: G.G. Birch (Editor). Analysis of Food Carbohydrate. Elsevier Applied Science Publishers, London

Meier U., 2001. Growth Stages of Mono- and Dicotyledonous Plants. BBCH Monograph. Federal Biological Research Centre for Agriculture and Forestry, Braunschweig. Online available at www.bba.de/veroeff/bbch/bbcheng.pdf, verified 08.03.2008

Menke K.H., Raab L., Salewski A., Steingass H., Fritz D., Schneider W., 1979. The estimation of the digestibility and metabolizable energy content of ruminant feedingstuffs from the gas production when they are incubated with rumen liquor in vitro. J. Agr. Sci. 93, 217-222

Menke K.H., Steingass H., 1988. Estimation of the energetic feed value obtained from chemical analysis and in vitro gas production using rumen fluid. Anim. Res. Dev. 28, 7-55

Naumann C., Basler R., 1976. Methodenbuch Bd III. Die chemische Analyse von Futtermitteln. Verlag Neumann, Melsungen

Pell A.N., Schofield P., 1993. Computerized monitoring of gas production to measure forage digestion in vitro. J. Dairy Sci. 76, 1063-1073

Shenk J.S., Westerhaus M.O., 1991. Population structuring of near infrared spectra and modified partial least square regression. Crop Sci. 31, 1548-1555

Stuth J., Jama A., Tolleson D., 2003. Direct and indirect means of predicting forage quality through near infrared reflectance spectroscopy. Field Crops Res. 84, 45-56

Tang S., Tan Z., Zhou C., Jiang H., Hiang Y., Sheng L., 2006. A comparison of in vitro fermentation characteristics of different botanical fractions of mature maize stover. J. Anim. Feed Sci. 15, 505-515

Theodorou M.K., Williams B.A., Dhanoa M.S., McAllan A.B., France J., 1994. A new gas production method using a pressure transducer to determine the fermentation kinetics of ruminal feeds. Anim. Feed Sci. Tech. 48, 185-197

Tolera A., Sundstøl F., 1999. Morphological fractions of maize stover harvested at different stages of grain maturity and nutritive value of different fractions of the stover. Anim. Feed Sci. Tech. 81, 1-16

Van Laar H., Van Straalen W., Van Gelder A.H., De Boever J.L., D'heer B., Vedder H., Kroes R., de Bot P., Van Hees J., Cone J.W., 2006. Repeatability and reproducibility of an automated gas production technique. Anim. Feed Sci. Tech. 127, 133-150 